\title{
Una visión (semi)heterodoxa de la crisis. Un simulacro de formalización parcial
}

\author{
Juan Urrutia \\ Catedrático de Fundamentos del Análisis Económico \\ Presidente del Patronato de la Fundación Urrutia Elejalde
}

\section{INTRODUCCIÓN}

En este trabajo trataré de exponer algunas ideas un sí es no es heterodoxas sobre lo que está pasando en la economía mundial en los últimos dos años y sobre lo que habría que hacer para salir de una situación como la actual, bastante comprometida. Espero cumplir con mi responsabilidad profesional de explicar, en mi caso desde un punto de vista (semi)heterodoxo, la naturaleza de la recesión generalizada y de la política económica recomendable para evitar la depresión. Todo ello junto con un intento muy rudimentario y parcial de modelar formalmente algunos aspectos de la crisis en una especie de simulacro teórico.

Comienzo por decir que la tarea no es fácil. En primer lugar porque estamos ayunos de teoría general reciente que nos permita enmarcar los problemas ${ }^{1}$ aunque los últimos años hayan sido pródigos en el desarrollo de modelos macrodinámicos con expectativas racionales, aunque contemos con explicaciones formales de aspectos parciales ${ }^{2}$ y aunque estemos ahogados por una información mediática abrumadora que distorsiona los hechos y exagera las cifras contribuyendo al pesimismo y quizá al pánico. La razón de esta abundancia desordenada es, posiblemente, la necesidad que tienen los medios de comunicación de competir con Internet que, por su parte, nos ofrece un océano de

1 No es el caso de Caballero et.al.(2008). Para una aproximación más sencilla y más dirigida ver las dos columnas de Caballero en VOXEU el 23 de enero de este año.

2 Véase Guerrieri y Kondor (2008). 
información, datos y, más recientemente, opiniones mucho más matizadas que las de los medios tradicionales ${ }^{3}$. La tarea se complica, en segundo lugar, por la «timidez» inicial de los economistas profesionales para dar a conocer sus ideas (a menos de que estén escritas en la forma técnica de «papeles» técnicos publicables en revistas especializadas y, por lo tanto, productivos para su carrera investigadora) lo que les ha llevado a estar en aparente silencio hasta muy recientemente. Solo hace dos meses, el 29 de diciembre, que salió el primer informe reseñable de FMI firmado, entre otros, por Blanchard, el economista jefe de esa institución y profesor del $\mathrm{MIT}^{4}$; y es (con la excepción de las columnas semanales de Krugman en el NYT) sólo desde hace tres o cuatro meses (desde la caída de Lehman realmente) que algunos economistas profesionales de la máxima reputación han comenzado a prodigarse como columnistas en diversos foros con notas no formalizadas y, en general, sobre aspectos parciales. Hasta ese momento, estábamos en manos de analistas de inversiones, muy respetables sin duda, pero con un punto de vista muy particular y de los servicios estudios de los Bancos Centrales, cuyos modelos de base del tipo macrodinámico mencionado, son más bien desconocidos en sus detalles por los externos a esas instituciones y, por lo tanto, de difícil evaluación, sin que, en consecuencia, podamos decir que contamos con suficientes trabajos técnicos sobre la forma de entender lo que está pasando. Y para terminar de dificultar la tarea hay que reconocer que, aunque todos los economistas mínimamente serios veían venir el pinchazo de la burbuja inmobiliaria, han sido muy pocos los que mostraron su preocupación de manera seria logrando la atención escéptica de la profesión y menos todavía los que hoy se atreven a predecir la profundidad o la duración de la recesión en un país o una zona económica determinada ${ }^{5}$.

Pues bien, ante una situación intelectual de este cariz, voy a incidir en algunos puntos que creo necesarios, o al menos convenientes, para entender la crisis y sus posibles remedios tratando de detectar las pautas que podría seguir un intento serio de formalización. En la primera sección trataré de explicar porqué creo que la «dependencia

3 Véase Economist.com, especialmente la mesa redonda formada por los comentarios a Blanchard de varios economistas, Vox EU y Sociedad Abierta. Esta última ha publicado los artículos de un e-book editado por FEDEA. Cabe mencionar también The Economist que ha dado la oportunidad a Blanchard de escribir un Economic Focus en el primer número de esta año 2009 y sobre el que pivota la mencionada mesa redonda.

${ }^{4}$ Véase Spillimbergo et al. (2008).

${ }^{5}$ Granger apelaba en su conferencia en la Carlos III del 19 de enero a la idea del Black Swan de N.N.Taleb (ver referencias) para justificar esa impotencia, apelación que en sí misma demuestra la perplejidad reinante. 
del recorrido», como fenómeno que enturbia la ciencia en general, es responsable de la perplejidad reinante y cómo podemos intentar introducir algunos ideas heterodoxas aunque sea a contrapelo. En la segunda sección intentaré introducir esas ideas heterodoxas que encuentro prometedoras en un modelo formal muy conocido, sencillo y perfectamente ortodoxo. Esto me permitirá, en la sección tercera, describir la situación precrisis y, en la cuarta, introducir el detonante de la crisis y presentar la situación actual. En la sección quinta examinaré las políticas que pueden ser utilizadas para salir de esa situación actual sin caer en la depresión introduciendo la noción del «período de ajuste» que puede ser tratada aquí, justamente, gracias a la rudimentaria modelización efectuada. En la sexta sección ofrezco algunos comentarios misceláneos sobre asuntos menos generales y más a ras de tierra añadiendo mi opinión sobre el futuro del Capitalismo. En la última sección resumo y concluyo.

\section{DEPENDENCIA DEL RECORRIDO Y HETERODOXIA}

En otro lugar ${ }^{6}$ he explicado la estrategia investigadora óptima, una estrategia rebelde y experimentadora, así como las razones por las que no la observamos en general y observamos en cambio «encasquillamiento» (lock-in effect) y «dependencia del recorrido» (path dependency). Es este último fenómeno el que me gustaría examinar en conexión con una cierta teoría macroeconómica fragmentaria y casi olvidada pero que, sin embargo, puede ayudar a entender correctamente la crisis. Por lo tanto voy a centrar mi atención en ciertas ideas teóricas que creo serían útiles en estos momentos pero que no reciben la atención debida por razones que intentaré explicar al final de esta sección.

Para intentar retomarlas es bueno, sin embargo, comenzar por un comentario aparentemente tangencial. Parece ser que finalmente la mayoría de los Estados están empezando a poner en práctica una política fiscal de gasto. Una posible pregunta es si llegan a tiempo. Quizá sea ya tarde, pero también podría ocurrir que sea demasiado pronto. El último premio Nobel, Paul Krugman piensa (y así lo ha dicho en numerosas columnas del NYT incluyendo la escrita inmediatamente después de aprobado el plan de Obama) que se ha perdido mucho tiempo y que el gasto público que se necesitaría para reactivar una economía como la estadounidense sería ya demasiado grande como

${ }^{6}$ En El Capitalismo que Viene (2008) he perfilado lo que entiendo es la estrategia investigadora optima. Una estrategia experimentadora y rebelde. 
para poder ser movilizado. Y también piensa, en consecuencia, que tenemos el peligro de caer en una situación como la japonesa de los años 90. Otros economistas no tan conocidos o con menor exposición mediática, como Kobayashi, al que me referiré más adelante, expresan su escepticismo ante estas medidas porque, según ellos, no serán efectivas a no ser que previamente las instituciones que componen el sector financiero hayan sido fregadas hasta hacerlas resplandecer como una patena de forma que el sistema de pagos en todas sus ramificaciones funcione sin fallos y sin temor a que éstos puedan surgir.

Pues bien, ahora me interesa explicar esta última opinión puesto que ello nos pone en contacto con esas ideas macroeconómicas que han sido arrumbadas y que sin embargo podrían haber sido esclarecedoras precisamente por haber sabido quedarse al margen del recorrido fijado para la ciencia económica. Para defender esta opinión me permito recordar lo que ya dije en otro lugar ${ }^{7}$. Proponía entonces que se explorara la idea heterodoxa de entender la situación como una forma de fallo de demanda efectiva del tipo que ocurre, según dice Axel Leijonhufvud en una entrevista del 2002 realizada por Snowdon (ver referencias), cuando es imposible poder decir de manera creíble algo de este tenor: «tengo este proyecto de inversión que rendirá sus frutos en el futuro y desearía intercambiar hoy esa perspectiva por factores de producción que me permitan producir hoy los bienes futuros». Y continúa: «y es ahí donde llegamos cuando el sistema financiero está totalmente atorado con préstamos malos», una forma de expresión que sienta como un guante a la situación actual. En estas circunstancias, en efecto, la atención se vuelve inmediatamente a la idea concomitante de un corredor neoclásico ${ }^{8}$ dentro del cual las señales de los mercados funcionan porque en su seno los precios son flexibles, pero fuera del cual nos encontramos con precios fijos que, al no transmitir información sobre la situación, generan unos ajustes en cantidades que originan exceso de capacidad instalada y paro en los sectores donde los precios y los salarios son efectivamente rígidos o no suficientemente flexibles.

Sin embargo, en este punto y antes de tratar de modelar esas ideas, quiero comentar porqué esta línea de pensamiento macroeconómico ha tenido poco eco y su utilización ha sido escasa, según reconoce Laidler ${ }^{9}$. Un par de detalles pueden ayudar a entender

7 Véase Urrutia ( 2008 a y 2008 b), dos columnas publicadas en febrero y marzo del año pasado en Expansión y recogidas en mi blog: juan.urrutiaelejalde.org.

${ }^{8}$ Véase A. Leijonhufvud (1973).

9 Laidler hizo este comentario en la laudatio de Axel Leijonhufvud pronunciada en el homenaje que UCLA rindió a este último. http://farmer.sscnet.ucla.edu/NewWeb/testing/AxelConfe- 
esta bifurcación fallida en el recorrido de la teoría macroeconómica. A principios de los años 70 las ideas de esta Macroeconomía del Desequilibrio ${ }^{10}$ competían con la llamada Síntesis Neoclásica y parecían hacer mella en esta última aproximación. Hasta tal punto que el libro de R. Barro y H. Grosman (1976 ), que seguía el planteamiento de precios fijos propio de la Macroeconomía del Desequilibrio, fue un libro utilizado como texto en cursos relativamente avanzados de Macroeconomía. Sin embargo treinta años más tarde el mismísimo Barro confiesa ${ }^{11}$ que le hubiera gustado no escribir ese libro. Ese rechazo retrospectivo se debía sin duda al establecimiento definitivo del modelo de equilibrio general competitivo como modelo estándar del pensar teórico puesto que en ese modelo estándar no hay lugar para el desequilibrio pues los precios son perfectamente flexibles. Es ese modelo el que conforma una base para la creación y desarrollo espectacular de la macroeconomía dinámica con expectativas racionales que acabó con cualquier otro intento de teorización ${ }^{12}$.

\section{Heterodoxia EN UN MODELO NEOCLÁSICO ELEMENTAL}

Trataré ahora de formalizar, al menos en parte, esas ideas heterodoxas que ya no circulan como ideas-guía a fin de contribuir a paliar esta escasez de modelos formales proponiendo una variante simple del más simple de los modelos de crecimiento (Solow 1956) que permite introducir dos ideas heterodoxas propias de la Macroeconomía del Desequilibrio, los fallos de demanda efectiva asociados al corredor neoclásico que, a mi juicio, resultan útiles para la comprensión de lo que pasa.

De hecho un artículo ya muy antiguo publicado en esta revista ${ }^{13}$ sería útil para entender la idea del corredor neoclásico y para discutir formalmente las posibilidades de la política monetaria. Al mostrar la existencia de una multiplicidad de equilibrios en un modelo de crecimiento neoclásico con dinero, es un precedente evidente del modelo de Dohtani, Inaba y Osaka (2007) que consiguen el mismo resultado de mul-

rence/Papers/Laidler.pdf.

10 Véase Leijonhufvud (1968).

11 Confesión que forma parte de una conversación personal que tuvimos en la cena que siguió a una charla académica celebrada en el IE de Madrid; pero para que se vea que no es una exageración mía, ver http://angrybear.blogspot.com/2009/01/barro-on-keynes-barro-and-grossman.html.

12 Véase Sargeant (2008) para reconocer el estado del arte en esta materia.

13 Véase Urrutia (1975) 
tiplicidad en un modelo similar al de Solow, pero en el que el consumo depende de la renta permanente. Sin embargo, y sin perjuicio de volver sobre ese tipo de modelos de crecimiento y dinero en otra ocasión, me parece más útil ahora comenzar por trabajar con el modelo más sencillo posible que, de momento, ni siquiera considera un mercado financiero. Consideraré que todos los bienes de consumo, todos los bienes de capital duradero y todos los otros activos han sido agregados en un solo bien que es producido por la tecnología existente y que todos los tipos de trabajo han sido agregados en uno solo. Tenemos por lo tanto, tres mercados: el del output, Q, con su precio, $\mathrm{P}$, que es al mismo tiempo el precio de los bienes de consumo y el precio de compra del capital físico u otros activos; el de los servicios de capital, $\mathrm{K}$, con su precio R, precio del uso de capital (o tipo de rendimiento de otros activos) y el de los servicios del trabajo, L, con su precio W. Los tres mercados son, en principio, de competencia perfecta y los precios se deberían ajustar inmediatamente hasta alcanzar el equilibrio. En cada momento de tiempo y mientras el sistema funciona sin sobresaltos las dotaciones de capital y trabajo se utilizan plenamente para producir el output que se consume y se ahorra. El ahorro se traduce inmediatamente en adición al capital existente (inversión).

Miremos a la tecnología. Supongamos una función de producción neoclásica estándar $\mathrm{Q}=\mathrm{F}(\mathrm{K}, \mathrm{L})$ con rendimientos constantes a escala de forma que el output per capita, $q$, las correspondientes productividades marginales $F_{K}$ y $F_{L}$, así como la relación marginal de sustitución, RMS, pueden escribirse como funciones de la relación capital/ trabajo denotada por k. Si además tomamos el precio $\mathrm{P}$ del output como numerario y suponemos competencia perfecta, la RMS se puede escribir como igual al precio relativo del trabajo y el capital:

$$
\begin{gathered}
q=f(k)=F_{K} k+F_{L} \\
F_{K}=f^{\prime}(k) \\
F_{L}=f(k)-k f^{\prime}(k) \\
R M S=\frac{f(k)-k f^{\prime}(k)}{f^{\prime}(k)}=\frac{(W / P)}{(R / P)}=\frac{W}{r}
\end{gathered}
$$

Estos conceptos están reflejados en el panel b) de la figura 1 en donde, dada la relación capital/trabajo OA, tenemos inmediatamente el output per capita, OC, la productividad marginal del trabajo, OD, la renta (per capita) del capital, CD y la relación marginal de sustitución OE. 
Figura 1. La Tecnología

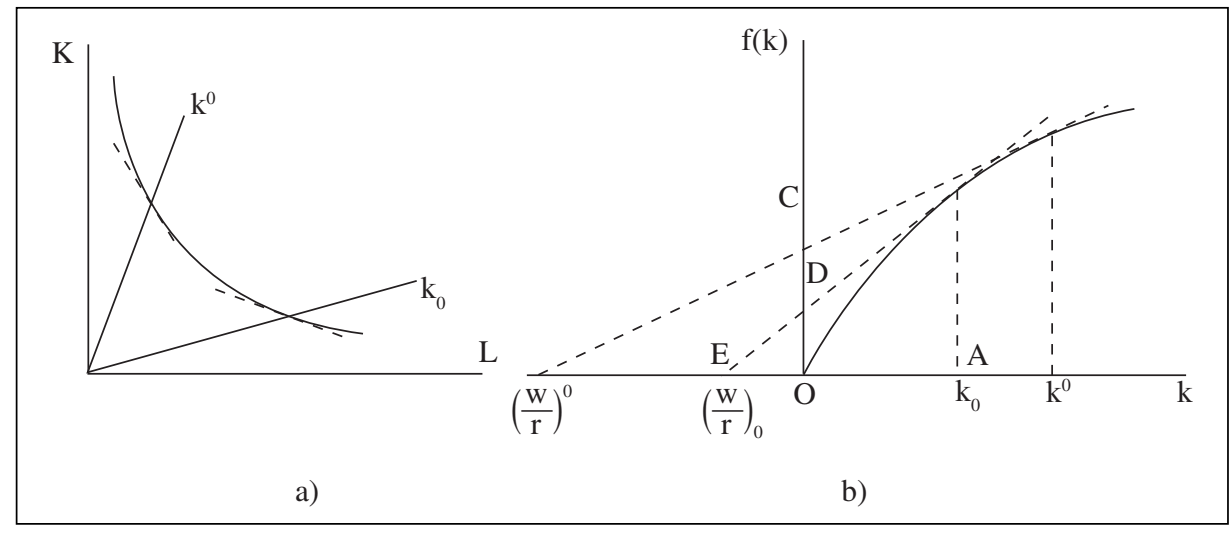

Notemos el hecho elemental que, a medida que disminuimos el capital y aumentamos el trabajo, relativamente hablando, la relación marginal de sustitución disminuye, es decir que para producir lo mismo se necesita cada vez más trabajo para compensar una caída dada del capital. Eso es lo que muestra el panel a) al pasar de $\mathrm{k}^{0}$ a $\mathrm{k}_{\mathrm{o}}$. Ahora bien si en una economía (como por ejemplo la española en los últimos años) se va cada vez más hacia actividades intensivas en trabajo como el turismo y la construcción, lo que debemos esperar es que la productividad marginal del capital aumente relativa a la productividad marginal del trabajo de forma que el precio de los servicios del capital suba en relación al precio de los servicios del trabajo.

Ahora bien, si los precios de los servicios del capital y de los servicios del trabajo, no se ajustan correspondientemente porque, por ejemplo, el salario nominal sigue subiendo en mayor proporción que la subida de los precios de los bienes de forma que el salario real aumenta más que el precio real de los servicios del capital, r, se genera desempleo. O, dicho de otra manera, si insistimos en mantener la participación del capital no hay más remedio que prescindir de trabajadores. Podríamos decir de manera no precisa que se mantienen los dividendos y se disminuye el empleo al no poder bajar los salarios nominales. Incidentalmente, esto responde a la pregunta popular de cómo puede entenderse que, el mismo día que se desploma el empleo, la Bolsa se recupera. He aquí el primer rasgo heterodoxo que quiero introducir en el modelo: la posible y relativa rigidez de precios, un rasgo éste que ya forma parte de cualquier de los modelos utilizados por casi cualquier Banco Central y a los que nos referíamos más arriba.

Para adaptar esa forma de entender el sistema económico a mis finalidades nos interesa entender el funcionamiento del ahorro (y del consumo) en un sistema con un 
sector público y con dos clases sociales, capitalistas y trabajadores. En consecuencia introducimos un sector público (que posee en propiedad un a\% del capital y que llamaré tamaño del sector público) y una diferenciación, en el sector privado, entre capitalistas y trabajadores. Evidentemente cambios en el tamaño del sector público cambian la distribución de la renta según nuestras definiciones; pero no hay porqué tener esto en cuenta porque la pérdida de renta de la clase capitalista ha de ser, en el modelo, sólo aparente, ya que el ingreso que reciben por el capital que pasa a manos del Gobierno ha de ser tal que les proporcione la misma renta que el capital traspasado proporciona al Gobierno.

Ahora podemos describir al sector privado. Los capitalistas poseen un (1-a)\% del capital existente y no trabajan. Reciben una renta $(\mathrm{Y})$, de $(1-\mathrm{a}) \mathrm{F}_{\mathrm{K}} \mathrm{K}$, pagan unos impuestos de $\mathrm{t}_{\mathrm{c}}(1-\mathrm{a}) \mathrm{F}_{\mathrm{K}} \mathrm{K} \mathrm{y}$, en consecuencia, su renta disponible es :

$$
\mathrm{Y}_{\mathrm{Cd}}=\left(1-\mathrm{t}_{\mathrm{C}}\right)(1-\mathrm{a}) \mathrm{F}_{\mathrm{K}} \mathrm{K} \quad 1 \geq \mathrm{t}_{\mathrm{C}}>0
$$

Los trabajadores no son propietarios del capital, reciben un salario $\left(\mathrm{Y}_{\mathrm{T}}\right)$ de $\mathrm{F}(\mathrm{K}, \mathrm{N})$ $\mathrm{F}_{\mathrm{K}} \mathrm{K}$, pagan unos impuestos de $\mathrm{t}_{\mathrm{T}} \mathrm{Y}_{\mathrm{T}} \mathrm{y}$, en consecuencia, su renta disponible es:

$$
\mathrm{Y}_{\mathrm{Td}}=\left(1-\mathrm{t}_{\mathrm{T}}\right)\left[\mathrm{F}(\mathrm{K}, \mathrm{N})-\mathrm{F}_{\mathrm{K}} \mathrm{K}\right], \quad 0<\mathrm{t}_{\mathrm{T}}<\mathrm{t}_{\mathrm{C}} \leq 1
$$

El sector público es muy fácil de describir ahora. El gobierno posee en propiedad un a\% del capital existente. Sus gastos vienen dados por la necesidad de mantener ese porcentaje. Sus ingresos provienen de los intereses sobre su capital y de los impuestos. En consecuencia, el superávit o déficit presupuestario es:

$$
\mathrm{D}=\text { Ingresos }- \text { Gastos }=\mathrm{a} \mathrm{F}_{\mathrm{K}} \mathrm{K}+\mathrm{t}_{\mathrm{C}} \mathrm{Y}_{\mathrm{C}}+\mathrm{t}_{\mathrm{T}} \mathrm{Y}_{\mathrm{T}}-\mathrm{a} \dot{\mathrm{K}}
$$

Podemos a continuación describir la acumulación de capital y el correspondiente modelo de crecimiento. Comenzando por la acumulación de capital diremos que el ahorro privado se efectúa de acuerdo con la teoría convencional del consumo, como una proporción de la renta disponible. Sea $\mathrm{s}_{\mathrm{C}} \mathrm{y} \mathrm{s}_{\mathrm{T}}, \mathrm{s}_{\mathrm{C}}>\mathrm{s}_{\mathrm{T}}$, las tasas de ahorro de capitalistas y trabajadores respectivamente. El ahorro total del sector privado viene dado por

$$
\mathrm{S}_{\mathrm{pr}}=\mathrm{S}_{\mathrm{C}}+\mathrm{S}_{\mathrm{T}}=\mathrm{s}_{\mathrm{C}} \mathrm{Y}_{\mathrm{Cd}}+\mathrm{s}_{\mathrm{T}} \mathrm{Y}_{\mathrm{Td}}
$$


Suponiendo que cualquier superávit presupuestario no es utilizado para modificar el tamaño del sector público, podemos afirmar que la acumulación de capital pública (igual al ahorro público) viene dada por:

$$
\mathrm{S}_{\mathrm{pu}}=\mathrm{a} \dot{\mathrm{K}}
$$

Ahora estamos en disposición de establecer un modelo de crecimiento; pero antes es necesario justificar la posible incongruencia para los trabajadores de ahorrar y no ser propietarios del capital. Es pues, necesario para la congruencia del modelo, suponer que su ahorro se mantiene en dinero líquido. Por la Ley de Walras el mercado de dinero está en equilibrio puesto que el de trabajo y el de bienes lo están en todo momento de tiempo. Notemos que esa ley no tiene porqué darse en caso de que los mercados no estén en equilibrio debido a la rigidez de algunos precios.

El modelo de crecimiento a largo plazo es muy fácil de obtener. Si la función producción es Cobb-Douglas, podemos definir una tasa global de ahorro $\mathrm{S}$ tal que:

$$
\left.\mathrm{S} \mathrm{F}(\mathrm{K}, \mathrm{N})=\mathrm{S}_{\mathrm{Pr}}=\mathrm{s}_{\mathrm{C}}\left(1-\mathrm{t}_{\mathrm{C}}\right)(1-\mathrm{a}) \mathrm{F}_{\mathrm{K}} \mathrm{K}+\mathrm{s}_{\mathrm{T}}\left(1-\mathrm{t}_{\mathrm{T}}\right)\right)\left[\mathrm{F}(\mathrm{K}, \mathrm{N})-\mathrm{F}_{\mathrm{K}} \mathrm{K}\right]
$$

de forma que, cuando la tecnología es Cobb-Douglas,

$$
\mathrm{S}=\mathrm{s}_{\mathrm{C}}\left(1-\mathrm{t}_{\mathrm{C}}\right)(1-\mathrm{a}) \pi+\mathrm{s}_{\mathrm{T}}\left(1-\mathrm{t}_{\mathrm{T}}\right)(1-\pi)
$$

El ahorro total es:

$$
\dot{\mathrm{K}}=\mathrm{S}_{\mathrm{pt}}+\mathrm{S}_{\mathrm{pu}}=\mathrm{S}_{\mathrm{C}}+\mathrm{S}_{\mathrm{T}}+\mathrm{S}_{\mathrm{pu}}=\mathrm{S} \mathrm{F}(\mathrm{K}, \mathrm{N})+\mathrm{a} \dot{K}
$$

de donde obtenemos:

$$
\dot{K}=\frac{\mathrm{S} \mathrm{F}(\mathrm{K}, \mathrm{N})}{(1-\mathrm{a})}=\varphi \mathrm{F}(\mathrm{K}, \mathrm{N})
$$

Y, en consecuencia

$$
\dot{K}=\varphi \mathrm{f}(\mathrm{k})-\mathrm{nk}, \quad 0<\varphi<1
$$


que es análoga a la del modelo de Solow y, por lo tanto, no plantea ningún problema de existencia, unicidad o estabilidad en el caso de la función Cobb-Douglas tal como refleja la figura 2 .

Figura 2. El modelo de crecimiento

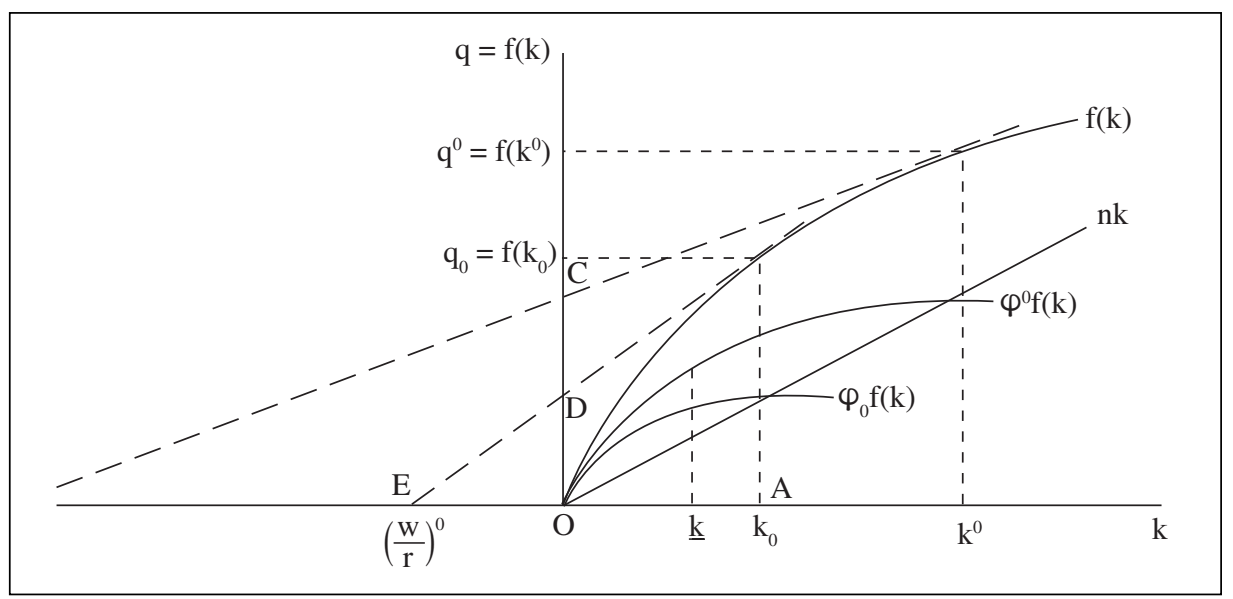

En esta figura 2 se ha reflejado también una relación capital/trabajo especial, denotada por $k$ y definida por

$$
\varphi \mathrm{f}^{\prime}(\mathrm{k})=\mathrm{n},
$$

que será de utilidad más adelante.

Notemos sin embargo que en general el equilibrio a largo plazo puede ser múltiple con un conjunto de equilibrios intercaladamente estables e inestables. Esto es así porque, a menos que las participaciones del capital y del trabajo sean constantes (como sería el caso si la función de producción fuera Cobb-Douglas) la derivada de la tasa de ahorro con respecto a la relación capital trabajo puede tener cualquier signo. En efecto, la tasa de ahorro viene dada por:

$$
\varphi(\mathrm{k})=\frac{\mathrm{S}}{(1-\mathrm{a})}=\frac{\mathrm{s}_{\mathrm{C}}\left(1-\mathrm{t}_{\mathrm{C}}\right)(1-\mathrm{a}) \prod(\mathrm{k})+\mathrm{s}_{\mathrm{T}}\left(1-\mathrm{t}_{\mathrm{T}}\right)(1-\mathrm{a}) \prod(\mathrm{k})}{1-\mathrm{a}}
$$


de forma que, como la derivada de $\Pi$ con respecto a $\mathrm{k}$ puede tener cualquier signo, lo mismo ocurre con la derivada de la tasa de ahorro con respecto a la relación capital/trabajo lo que no se reconoce en el artículo original de Solow (Solow (1956)) y, lo que es más relevante, su relación con n puede ser cualquiera tal como sí reconoce ese artículo original, de modo que en la figura correspondiente la curva denotada por $\varphi \mathrm{f}(\mathrm{k})$ puede estar por encima o por debajo de la recta nk.

Figura 3. El corredor neoclásico

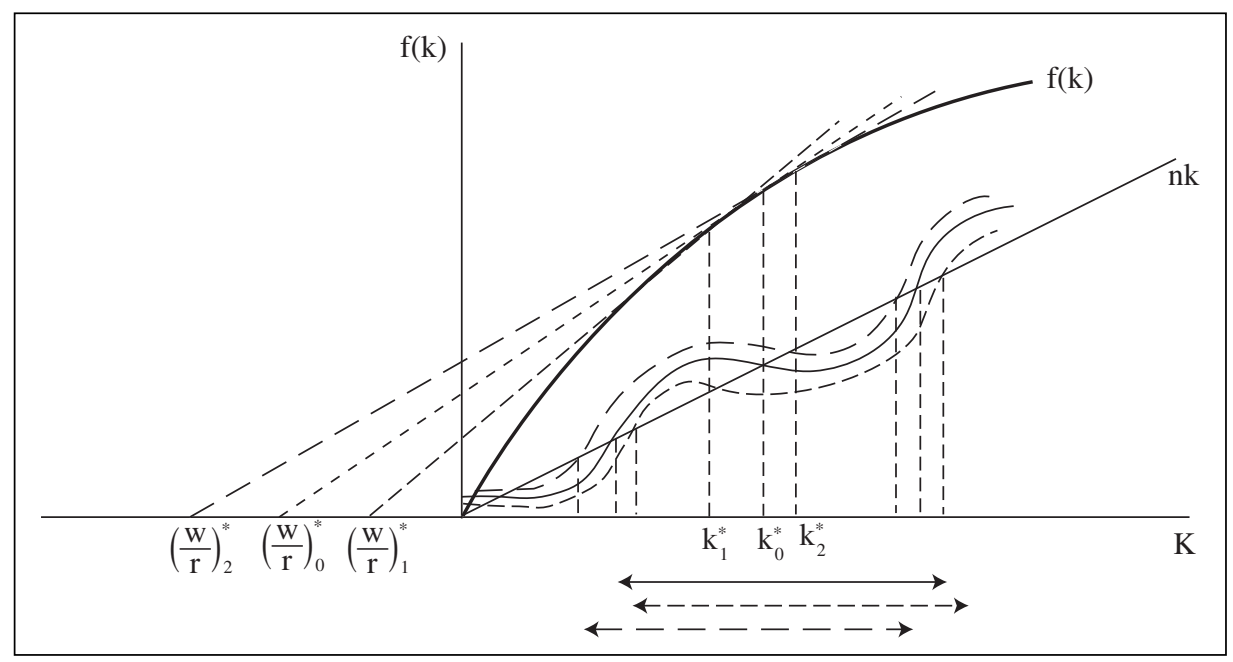

La figura 3 refleja el modelo de crecimiento y sus posibles equilibrios. En ellas se refleja con claridad el segundo toque de heterodoxia que quiero introducir : la existencia de un corredor neoclásico dentro del cual la $\mathrm{k}$ de equilibrio es estable y fuera del cual la $\mathrm{k}$ a la que nos lleve un posible shock no es estable. Esta figura 3 será de utilidad más adelante para entender el detonante de la crisis y sus posibles soluciones.

\section{LA SITUACIÓN PREVIA A LA CRISIS}

En el contexto del modelo anterior voy a tratar de pensar en una economía global y única. Por lo tanto dejaré para más adelante los problemas particulares de la economía española y todos los aspectos que tienen que ver con una economía abierta a otras como son los déficits exteriores, el desacoplamiento y el posible proteccionismo. 
Imaginemos por lo tanto un sistema económico como el descrito en su trayectoria estacionaria creciendo a un 3\% anual en términos reales. Aunque no esté modelada imaginemos que dicho sistema muestra una inflación del 2\% gracias a una política monetaria razonable y, he aquí la novedad no modelada formalmente, con un endeudamiento creciente tanto del sector público (debido a la factura energética cuyo incremento es asumido en parte por el sector público sin incrementos en las tasas impositivas) como del sector privado debido a ese mismo incremento del precio del petróleo, a la existencia de una burbuja inmobiliaria y a una gran innovación financiera.

Hay una burbuja inmobiliaria por muchas razones, ente las cuales merece la pena mencionar los bajos tipos de interés (por debajo del tipo de interés «natural» wikselliano) engendrados tanto por una política monetaria anterior que tuvo que frenar varias amenazas al crecimiento bajando los tipos como por la demografía que, por un lado, propicia el retiro, quizá en sitios distintos a los del lugar de trabajo y residencia habituales y, por otro lado, fuerza los movimientos migratorios que exigen creación de vivienda nueva.

Y hay también una enorme innovación financiera que acomoda la burbuja creando nuevos instrumentos derivados como los CDO's (Collateralized Debt Obligations) o CDS's (Credit Default Swaps) relacionados respectivamente con la titulización y con el aseguramiento de nuevos riesgos, así como nuevos productos estructurados que representan más bien una innovación organizativa y contable. Me refiero a los $\mathrm{ABCP}$ (Asset Backed Commercial Paper). Una nueva entidad, financiada y asegurada en parte por un banco comercial que carga sus fees y denominada conduit, compra facturas de diferentes productores de lo que sea proporcionándoles liquidez y a su vez genera papel comercial para seguir haciendo lo mismo sin que el banco organizador tenga que reflejar la financiación inicial en sus libros.

En este punto quizá sea razonable dejar constancia que el origen próximo del problema en el que nos encontramos está en la política de tipos bajos que siguió Greenspan para solucionar algunas crisis previas ${ }^{14}$ y que obligó a los bancos a hacer lo que no estaban acostumbrados a hacer: trabajar con tipos bajos. Para seguir obteniendo beneficios tuvieron que aumentar la cantidad «vendida» y para ello se vieron impelidos hacia el diseño y la colocación de nuevos productos derivados y de nuevas hipotecas y a titulizar muchas de estas inversiones para poder financiarse a partir del activo, aumentando así su deuda en relación a su capital.

14 Bastará mencionar aquí la crisis de los Savings and Loan Associations, la asiática y sus reverberaciones en Rusia y Méjico, la crisis de LTCM y, más recientemente la que siguió al pinchazo de la burbuja de las puntocom. 
Merece la pena detenerse brevemente en la naturaleza de esa deuda flotante a fin de salir al paso de no pocas ideas incorrectas que surgen en casos como el actual. Esta deuda flotante tiene naturaleza de inside money ${ }^{15}$. Un derivado financiero es solo una apuesta entre dos personas de forma que cuando una gana la otra pierde. Así de golpe esto parece querer decir que la cantidad global de esas apuestas es irrelevante. Pero cada una de estas personas que se ha arriesgado en una apuesta en forma de derivado financiero puede asegurarse contra la contingencia que le es desfavorable con una o dos o... $\mathrm{N}$ «terceras personas» que, a su vez, no hacen sino apostar y así. ... ad infinitum. ¿Sigue siendo cierto que el monto total de la riqueza en juego es irrelevante? Si en un momento determinado la cadena descrita se cierra y los primeros apostantes aseguran a los últimos, hay una inmensa nube flotante de deudas sociales que al cancelarse unas con otras valen cero en neto. Veríamos redistribución de recursos, pero no creación neta de riqueza en el sentido habitual con el que se usa esa palabra en Macroeconomía aunque, desde luego, observaríamos oportunidades antes no existentes y una mejor asignación del riesgo, cosas estas que debieran considerarse como parte de la riqueza. Reverdeciendo nociones de teoría monetaria podríamos decir que esa «nube flotante» es inside money que «solo» sirve para facilitar los intercambios, dar fluidez al crédito y dejar alguna ganancia neta a algunos y pérdidas netas a otros. Su cantidad no es irrelevante y de hecho podríamos decir que está relacionada con la anchura del corredor neoclásico aunque eso no se puede reflejar formalmente en nuestro modelo. ¿Y si no se cierra la cadena? Entonces puede haber creación de riqueza neta en el sentido tradicional junto con un riesgo que gira contra esa riqueza y es tanto más grande cuando más larga es la cadena, con independencia de las nuevas oportunidades y la mejor asignación del riesgo. Esta era justamente la situación inicial de la economía mundial antes del detonante de la crisis y, en una situación así, hay que tratar de establecer un compromiso adecuado entre riqueza y riesgo que no parece fuera el que se alcanzó en la situación previa la crisis.

\section{EL DETONANTE DE LA CRISIS Y LA SITUACIÓN ACTUAL}

Ahora estamos en disposición de introducir en nuestro modelo simple el detonante de la crisis. Se trata del pinchazo de la burbuja inmobiliaria. Recordemos que la burbuja existe porque, dada la previsible gran demanda ya mencionada, los ahorradores creen hacer un gran negocio comprando para revender. Esta demanda extra aumenta el

15 Véase Pesek y Saving (1967). 
precio de la vivienda y hace buenas sus expectativas lo que les lleva a hacer la misma operación a crédito, cosa que viene muy bien a los bancos que, ante los tipos bajos y tal como se acaba de decir, tienen que aumentar el volumen de negocio, generando nuevas hipotecas, amén de otros nuevos activos derivados o productos estructurados, a fin de seguir manteniendo los beneficios.

Cualquier observador y todo economista piensa que una situación así es insostenible pues nada aumenta de valor constantemente. Muchos amenazan con que viene el lobo, pero pocos son escuchados. Pero el lobo viene un día y lo hace en forma de la primera hipoteca impagada. A partir de ahí los especuladores inmobiliarios se dan cuenta de que las condiciones que sostenían sus expectativas ya no se dan y comienzan a retirar su demanda de crédito. En consecuencia los bancos se ven forzados bien a reducir beneficios bien a mejorar las condiciones de las hipotecas rebajando la calidad de su balance. Para mantener esa calidad, dado su capital, los bancos comienzan a ser remisos en la concesión de créditos con lo que, con un cierto retardo, la demanda de bienes de inversión acabará disminuyendo aunque de momento continúa la escalada de los precios de la energía. Nos encontramos pues con una situación desconcertante de crisis solapadas: la inmobiliaria, la de demanda agregada efectiva provocada por le pinchazo de la burbuja inmobiliaria y la ya olvidada crisis de oferta agregada correspondiente al alto precio de la energía. Ante una coyuntura de esta naturaleza los responsables de la política económica no saben qué hacer. Si subir o bajar los tipos ${ }^{16}$, si rebajar impuestos o aumentar el gasto o si no hacer nada de eso.

Pero a partir de este punto las cosas se van aclarando paulatinamente y haciéndose más familiares y reconocibles. El detonante descrito puede interpretarse y representarse en la figura 3. En esta figura, no fácil de «leer», se representa, en primer lugar y en trazo continuo, el sistema en equilibrio a largo plazo, $\mathrm{k}_{0}{ }^{*}$, con su asociado corredor neoclásico (la distancia entre los dos equilibrios inestables a derecha e izquierda de ese equilibrio inicial) correspondiente a una inicial tasa de ahorro previa a la crisis que refleja la muy alta demanda de inversión. Pero también se representa, en trazo discontinuo corto, la caída generalizada de la tasa de ahorro causada por el detonante de la crisis y que nos lleva a colocarnos dentro de un nuevo corredor neoclásico de forma que el sistema camina hacia un nuevo equilibrio a largo plazo, denotado por $\mathrm{k}_{1}{ }^{*}$. Es posible y deseable que el sistema tienda hacia ese nuevo equilibrio él solo; pero puede ocurrir que, si la caída de la demanda de inversión ha sido muy grande, la dinámica le lleve hacia el origen, caso éste en el que podríamos decir que nos encaminamos a la depresión. Hagamos notar que, aunque este último no sea el

${ }^{16}$ La FED y el ECB hicieron lo contrario durante meses. 
caso, a medida que el sistema camina dentro del nuevo corredor neoclásico hacia el nuevo equilibrio los precios deberían ajustarse y que si no lo hacen observaremos, tal como ya se ha explicado, crecientes tasas de desempleo debidas precisamente a la resistencia de los precios a ajustarse en la dirección y magnitud que permitiría mantener el empleo. A efectos posteriores la figura 3 también refleja, en trazo discontinuo largo, el posible incremento de la demanda agregada efectiva conseguida mediante una política fiscal agresiva y que nos llevaría a un tercer equilibrio a largo, $\mathrm{k}_{2}{ }^{*}$, con su corredor neoclásico asociado.

No es difícil ahora imaginar cómo se llega en pocos meses desde esa situación desconcertante que sigue al detonante hasta la situación justamente anterior a la reunión del G-20+1 en Washington. La reducción del crédito y el empeoramiento de las expectativas reducen la demanda energética y de materias primas y se desploma el precio del petróleo. Se acabó la crisis de oferta. Ya no se hable de los años setenta, sino de la Gran Depresión y con mayor propiedad de la década perdida en Japón.

Nos quedamos pues con la crisis inmobiliaria y la consecuente crisis de demanda agregada efectiva. Respecto a la primera habría que hacer distinciones finas entre países y examinar con cuidado las escasas estadísticas serias disponibles. En mi opinión, respaldada en parte por Arellano y Bentolila (2009) en lo que a los precios se refiere, los precios no se ajustan con suficiente rapidez mientras la cantidad de viviendas de segunda mano no intercambiadas en el mercado y el parque de viviendas construidas y no vendidas aumentan notablemente con el resultado inmediato de un aumento significativo del paro entre los trabajadores de poca cualificación, en general inmigrantes, debido al frenazo de las nuevas promociones inmobiliarias. Las non performing loans alcanzan cotas alarmantes y los bancos cierran el grifo del crédito, los unos porque no tienen liquidez debido a que no les devuelven lo prestado, los otros porque ya han visto lo que viene y no quieren correr la misma suerte.

Mención aparte merece el mercado interbancario que se seca de golpe, antes de que puedan reaccionar los Bancos Centrales, porque ningún banco se fía de la solvencia de su colega lo que es muy racional dada la situación propia. Solo cuando estos Bancos Centrales comienzan a reaccionar proveyendo de liquidez se empieza a mover el mercado interbancario y a reducirse el correspondiente tipo de interés.

$\mathrm{Y}$ aquí estamos, en plena crisis de demanda agregada efectiva con un incremento serio del paro y con un mercado interbancario en recuperación pero todavía con spreads muy altos, con ajustes de cantidad ante la inflexibilidad de los precios que, aunque no general, es significativa en sectores importantes como la construcción, el automóvil y el crédito bancario. Esto es así bien porque, en los dos primeros casos, se resisten los promotores de viviendas y productores de automóviles quienes se agarran desesperadamente a unas expectativas desfasadas y, en el tercer caso, porque el mer- 
cado interbancario es todavía muy estrecho. La situación está cambiando en los dos o tres últimos meses y parece que los precios de la vivienda flexiona, aunque no al mismo ritmo que la cantidad (se mida esta como se mida), que el interbancario se reduce consistentemente aunque lentamente y que nada significativo ocurre con los precios de los automóviles.

\section{POLÍTICAS ECONÓMICAS A PARTIR DE LA SITUACIÓN ACTUAL}

Y a partir del reconocimiento de en dónde estamos comienza el capítulo de la discusión sobre las medidas a tomar para frenar el deterioro. Distinguiré entre políticas macroeconómícas tradicionales y las sectoriales y añadiré, aquí y allí, comentarios sobre la duración de la crisis.

\subsection{Políticas macroeconómicas tradicionales}

Comencemos por la Política Monetaria. El primer comentario obligado está relacionado con la perplejidad ya mencionada. Mientras la FED reconoció la naturaleza de demanda de la crisis y comenzó muy pronto a rebajar los tipos de intervención a los cuales los bancos pueden reponer su liquidez, el BCE pensó que seguíamos con una crisis de oferta y, en mi opinión, tardó demasiado tiempo en comenzar a bajarlos.

El segundo comentario obligado es que también se tardó demasiado tiempo en reconocer que la crisis financiera era de posible solvencia y no sólo de liquidez y que esta crisis de (in)solvencia estaba basada en la creencia por parte de cada banco de que el colega estaba como él, demasiado apalancado y con un activo de poca calidad. Lo que había que hacer es facilitar que el interbancario comenzara a funcionar después del consiguiente parón inicial y de la posterior ralentización; pero para ello había que, no solo continuar reduciendo el tipo de intervención, sino sobre todo eliminar la desconfianza y suministrar fondos a los bancos para mejorar la ratio capital/ deuda.

Esto último se ha hecho y se sigue haciendo, pero contra la opinión política de mucha prensa y, que yo sepa, sin la utilización explícita de un mecanismo que permita la autoselección de los diferentes bancos. Sospecho que los Bancos Centrales han fallado en lo que se suponía era su fuerte: su competencia intelectual. A partir de un momento dado han hecho cualquier cosa que permitiera la fluidez del crédito; pero lo reseñable, y este es el comentario más triste, es que a partir de ahora su 
credibilidad y su independencia están en entredicho con la posible excepción del Banco de España. No porque hayan permitido algunos experimentos desreguladores, sino porque no han sabido mantener su independencia y han sido capturados tanto por los Gobiernos como por el propio sector financiero, reaccionando como chiquillos cuando, como en el caso de Lehman, han intentado mantener su reputación dejándolo caer.

No parece que esta Política Monetaria tenga muchos más resortes aunque ciertamente el poder de un banquero central es tan grande que puede inyectar liquidez a cambio de casi cualquier colateral.

Continuemos con la Política Fiscal. Aquí también se ha tardado demasiado tiempo en reconocer que el precedente interesante era la década perdida de Japón. A partir de ese punto, se ha pretendido ignorar (o rebatir con argumentos serios) que el multiplicador de las rebajas impositivas según estudios fiables, es mayor que el multiplicador del gasto ${ }^{17}$ y se ha argumentado, correctamente en mi opinión, que el temido crowding out (o efecto expulsión) no opera en las circunstancias de crisis de demanda agregada efectiva en las que nos encontramos. Frente a la ortodoxia vigente hasta hace bien poco se argumenta que si el gasto público se lleva a cabo controladamente y en sectores productivos la deuda pública incrementada no tiene porqué recaer con todo su peso sobre los hombros de las siguientes generaciones que, desde luego, tendrán que pagarla, pero a partir de una renta considerablemente mayor. En cualquier caso la sugerencia de crear una Agencia Fiscal independiente con las características de un Banco Central no ha tenido demasiado eco quizá por la merma reputacional de la «agencia de agencias», el Banco Central, tan fácilmente capturable según se ha visto ${ }^{18}$.

Pues bien, ahora podemos aprovechar el simulacro de teoría que he efectuado en la segunda sección. Si, a pesar de que la batería de posibles medidas monetarias puede no esta agotada pues cabe que los Bancos centrales acepten casi cualquier cosa como colateral para proporcionar liquidez al sector bancario, los que creemos que lo relevante en este momento es la política fiscal deberíamos ser capaces de aportar algo distinto y nuevo a la discusión entre los defensores de rebajar impuestos y los defensores de

${ }^{17}$ Los comentarios en la Red sobre el tamaño de los multiplicadores son continuos. Baste con un ejemplo, el de Mankiew (2008).

18 Quizá como mera curiosidad pueda mencionar aquí que, en un trabajo antiguo ( Urrutia (1997) reproducido parcialmente en Urrutia (2008), se muestra cómo un juego entre dos agencias independientes como un Banco Central y una Agencia Fiscal, el óptimo en términos de inflación y crecimiento sólo se alcanzará si una y otra renuncian al «fanatismo». 
aumentar el gasto público, algo que fuera más allá o en otra dirección que la discusión mencionada sobre multiplicadores. Es en este punto en el que la modelización resulta ser útil.

Déjenme suponer ahora que, a pesar de que he rechazado imponer a priori la fijeza de las participaciones del capital y del trabajo, la función de producción alrededor de la relación capital/trabajo de equilibrio es del tipo Cobb-Douglas, una suposición que no me parece muy distorsionadora localmente hablando. En esas condiciones podemos tratar de intervenir en la discusión haciendo jugar la duración del período de ajuste, una noción que aunque antigua resulta hoy sorprendente por olvidada. Deberíamos inclinarnos por la forma de la política fiscal que nos llevara en menos tiempo desde la situación actual hasta las cercanías de un nuevo equilibrio a largo plazo prefijado y que también aparece representado en la figura 3. Pues bien, los resultados que conocemos son como sigue de acuerdo con Urrutia (1982) y Urrutia (1984).

Para empezar sabemos que, dentro de nuestro modelo, la tasa de ahorro generalizada que hemos utilizado, aumenta con un incremento en el gasto público que aumenta el tamaño del sector público y que también puede aumentar con una disminución de la tasa impositiva que disminuiría dicho tamaño. Pero la influencia de cambios en el gasto o en los impuestos en el período de ajuste no es algo tan evidente. Los resultados que conocemos exigen distinguir entre diferentes sistemas económicos que, aunque tengan acceso a una tecnología similar, se encuentren hoy en situaciones distintas en relación a la relación capital/trabajo denotada por $k$ y dibujada en la figura 2 . En efecto, el resultado central de Urrutia (1984), en donde pueden consultarse las pruebas que a su vez remiten a Urrutia (1982), dice que:

Sea $\mathrm{k}_{0}<\underline{\mathrm{k}}$

$$
\frac{\delta \mathrm{T} \alpha}{\delta \mathrm{a}}>0, \quad \frac{\delta \mathrm{T} \alpha}{\delta \mathrm{t}}<0
$$

Sea $\mathrm{k}_{0}>\underline{\mathrm{k}}$

$$
\frac{\delta \mathrm{T} \alpha}{\delta \mathrm{a}}<0, \quad \frac{\delta \mathrm{T} \alpha}{\delta \mathrm{t}}>0
$$

Resultados estos en los que a es el tamaño del sector público, t es la tasa impositiva, supuesta aquí común para ambas clases sociales y $\mathrm{T}_{\alpha}$ es el tiempo necesario para 
alcanzar el $\alpha \%$ de la relación capital/trabajo del equilibrio que se quiere alcanzar con la política fiscal aplicada.

La interpretación sería como sigue. Tomemos sistemas económicos denominados «ricos», en el sentido preciso de que, a pesar del detonante, $\mathrm{k}>k$. En estos países el $p e-$ ríodo de ajuste es menor cuanto mayor es el tamaño del sector público y cuanto menor es la presión impositiva, todo ello dentro de un aceptable «equilibrio» presupuestario que el lector puede verificar que puede obtenerse. Este resultado justificaría el planteamiento de la nueva administración americana, pero lo haría por razones que no tienen nada que ver con las esgrimidas hasta ahora en ese caso.

Naturalmente lo contrario ocurre en sistemas económicos «menos ricos», o emergentes, en el sentido de que $\mathrm{k}<k$. En estos sistemas el período de ajuste es menor cuanto menor sea el tamaño del sector público del que partimos y cuanto mayor sea la presión impositiva, dentro de un aceptable equilibrio presupuestario.

Además también podemos recordar otro resultado que relaciona el período de ajuste con un índice de desigualdad, d, interpretado como directamente relacionado con la redistribución hacia los ricos. Este resultado dice que:

$$
\begin{array}{ll}
\text { Sea } \mathrm{k}_{0}<\underline{\mathrm{k}}, \text { entonces } & \frac{\delta \mathrm{T} \alpha}{\delta d}>0 \\
\text { Sea } \mathrm{k}_{0}>\underline{\mathrm{k}}, \text { entonces } & \frac{\delta \mathrm{T} \alpha}{\delta d}<0
\end{array}
$$

Este segundo resultado puede interpretarse como diciendo que, en esos países que llamamos «ricos», el período de ajuste será tanto más breve cuanto mayor sea la desigualdad en el tratamiento fiscal en el sentido de mejorar a las rentas altas frente a las bajas. Parte de la ventaja de los EEUU sobre otros países desarrollados sería justamente su desigualdad inicial. Ni que decir tiene que en países que denominaríamos «menos ricos» o emergentes se dan los resultados contrarios. Así Brasil, por ejemplo, saldría de la crisis con menor presteza que otros países emergentes debido a su desigualdad inicial. Notemos que esta simetría entre países parecería algo bueno, pues eso puede llevar a que ciertos países se encuentren preparados antes para «tirar» de los demás. 


\subsection{Políticas sectoriales}

Cuando una crisis seria llega, los grupos de presión se ponen en funcionamiento y no pocos gobiernos se ven forzados a tomar algunas medidas sectoriales, como por ejemplo a favor del sector del queso parmesano, tal como ha ocurrido en Italia. Pero hablando un poco más en serio, podemos pasar revista a algunas políticas sectoriales que se han tomado o se medita tomar.

El mercado de trabajo es el primero que ha saltado a la rampa de lanzamiento impulsado por las asociaciones empresariales. Junto a recomendaciones muy sensatas (como la descentralización de la contratación colectiva) aparecen, como siempre, las ideas de flexibilizar el despido queriendo decir simplemente abaratar su coste. Esta medida así como la también demandada de reducir el «impuesto al empleo» o cuotas empresariales a la Seguridad Social, son materias demasiado serias y delicadas como para tratarlas al rebufo de una crisis o como tema de discordia entre un Gobierno y un Banco Central. Exigen una meditación muy pausada y relacionada con la innovación de forma que no puede despacharse con cuatro tópicos sobre la libertad en algún país anglosajón o sobre la «flexeguridad» nórdica o con citas oportunistas de Keynes como las que trae a colación Mario Rizzo (2009). Hay que debatir esta reforma estructural y hacerlo en serio. Para ello contamos con los trabajos referenciados de Arellano y Bentolila (2009) y de Felgueroso y Jiménez (2009). Pero también habrá que prestar atención a argumentos especiales en una época de deflación como el de Paul de Grauwe (2009) quien nos hace ver las posibles ventajas de las denostadas rigideces.

Por otro lado no creo que haya nadie serio que defienda que hay que apoyar al sector inmobiliario de manera específica ni siquiera para sostener indirectamente a aquellas instituciones financieras que se han pasado en su concesión de créditos hipotecarios o de créditos-promotor y que ahora ven crecer la mora. Y casi lo mismo cabe decir del sector del automóvil que como tal sector no está sufriendo tanto globalmente aunque lo esté pasando muy mal en algunos países.

¿Y qué decir del sector financiero, el foco del incendio? Como su limpieza es fundamental para que cualquier otra medida sea efectiva este parece el momento para reclamar el apoyo intelectual de ciertos economistas. Es precisamente el diseño de un arreglo institucional excepcional en el sector financiero lo que esperaríamos de los especialistas en Economía de la Información y/o en Diseño de Mecanismos. Para acercamos siquiera un poco a este menester exploremos las dos ideas complementarias debidas a Spence y Stiglitz quienes compartieron el Nobel con Akerlof en el 2001. Spence (1974) es el inventor del signalling: un banco que ha de financiarse para continuar con su negocio crediticio 
en un momento de asimetría informacional y de recelo ha de «señalar» que es solvente y merecedor de crédito mediante una señal que puede consistir, por ejemplo, en salir de compras porque en mala situación será irracional. Stiglitz (1975) es el perfeccionador del screening: el regulador del sector financiero diseña un plan de ayudas de tal naturaleza que funciona como un filtro en el sentido de que su uso revela la situación del banco pues los bancos se autoseleccionan dando origen a un equilibrio de los que denominábamos separador. Solo un arreglo que combine un screening inteligente que propicie las señales adecuadas puede lograr la limpieza del sector financiero.

Esta política sectorial es crucial pues la política fiscal de gasto público, hoy comúnmente admitida, no serviría de nada si no se ha limpiado previamente el sector financiero que debe canalizarla y no se ha puesto en marcha de nuevo el mercado interbancario. Aduzco una cita del ya mencionado profesor Kobayashi traducida libremente: «Una gran lección del Japón de los años 90 es que las políticas keynesianas per se no contribuyeron a la solución de la crisis financiera. Mientras que Japón instrumentó repetidamente grandes paquetes de estímulos fiscales, el gobierno no realizó un esfuerzo serio de política para que los bancos se deshicieran de sus préstamos fallidos. Como resultado un enorme montón de esos préstamos fallidos se inflamó e hinchó bajo la colusión implícita entre el gobierno y los bancos. Naturalmente la incertidumbre de los pagos... persistió durante años. El problema esencial era... que las políticas centradas en los trabajos públicos y en los recortes impositivos no eran lo suficientemente directas como para atacar el problema...» ${ }^{19}$.

5.3. Como enlace con la siguiente sección, conviene terminar ésta con un comentario breve y general sobre la duración y el tamaño de la crisis. Pues bien, más allá de los resultados ya mencionados en relación con la duración del período de ajuste para recuperar la situación inicial, poco se puede decir sobre esas dos cuestiones salvo que están íntimamente relacionadas y que ambas dependen del reconocimiento de la verdad que contienen las palabras citadas de Kobayashi. Si acabamos por aceptar que la

19 Véase Kobayashi (2008 b).El 13 de febrero Leijonhufvud dice algo que va exactamente en la misma dirección: «Fiscal stimulus will not have much effect as long as the financial system is deleveraging. Even if that problem were to be more or less solved, the government deficit would have to offset both the decline in industry investment and the rise in household saving - a gap that is rising as the recession deepens. Here, too, the public is sceptical and prone to conclude that a program that only slows or stops the decline but fails to «jump start» the economy must have been a waste of tax payers' money. The most effective composition of such a program is also a problem». Ver Leijonhufvud (2009). 
inflexibilidad de precios es crucial y está en el origen de esta crisis habremos de reconocer que cuanto antes se deje el sector financiero como los chorros del oro tanto más rápidamente se recuperara el mercado interbancario, volviendo a spreads «normales», cosa que ya está ocurriendo. Es pues razonable esperar que la demanda en aquellos sectores más afectados vaya volviendo a sus niveles anteriores comenzando así una recuperación sobre bases sólidas que se reflejará en las Bolsas del mundo y acabará por doblegar el pesimismo de las expectativas. La ventaja de esta diagnosis no está en su precisión sino en la apuesta por una terapia que, justamente, estaría en las manos de esos economistas versados en diseño de mecanismos. Les toca pechar con la ingeniería pertinente para apuntalar el edificio y no limitarse a estar agazapados para cuando toque volver a regular los mercados.

\section{Comentarios MisceláneOS}

Hasta este punto creo haber contribuido siquiera modesta y parcialmente a la comprensión de la crisis actual, su naturaleza, su punto de partida y sus posibles soluciones. Pero lo he hecho a partir de una especie de modelo (semi)heterodoxo y de carácter muy general que no presta atención a problemas concretos ni tampoco a los propios de economías abiertas. Es hora de remediar algunas de estas carencias aunque no pueda hacerlo más que de una manera muy impresionista.

El primer comentario que viene a la mente en cuanto pensamos en una economía nacional dentro del concierto de las naciones es el de la posible actitud nacionalista que lleva a algunos países al proteccionismo. He aquí una especie del dilema del prisionero. Todo el mundo sabe que la crisis se superaría antes si continuáramos con los esfuerzos por mantener abiertas las economías, pero nadie parece estar dispuesto a hacer el esfuerzo necesario para que esto ocurra. Todos acabaríamos mejor si evitáramos el proteccionismo y continuáramos con la liberalización del comercio mundial; pero las presiones de grupos de interés nacionales pueden ser lo suficientemente fuertes y pesadas como para que los gobiernos cedan ante ellas, se quiebre la tendencia a la liberalización y se ralentice la ronda de Doha o se paralice hasta nuevo aviso. Pero en circunstancias especiales, y las actuales lo son, deberíamos prestar oídos a argumentos de óptimo subsidiario que nos llaman la atención sobre el hecho, especialmente relevante para Europa, de que es posible que la demanda agregada efectiva global aumentara más si dejamos de tratar de aprovecharnos del incremento del gasto público del vecino, cerramos las fronteras e incrementamos nuestro gasto público nacional. 
Continuando en el contexto realista de economía abierta es el momento de volar más bajo y de ser más específico en lo que respecta a nuestro entorno más inmediato, sea Europa, sea España.

En cuanto a Europa, y más allá de lo que acabo de mencionar respecto al proteccionismo, hay que recalcar que la idea del desacoplamiento se ha derrumbado junto con la caída generalizada de la demanda agregada efectiva. El contagio es ya siempre global y lo único teóricamente serio que se me ocurre decir es que la probabilidad de contagio (financiero) es siempre menor y sus efectos menos perniciosos cuanto mayor es la «promiscuidad», es decir cuanto más diversificada esté una zona económica en sus relaciones comerciales y financieras con otras zonas ${ }^{20}$. Por otro lado el carácter confederal de Europa hace muy difícil la coordinación y ésta sí que es importante cuando se está hablando de gasto público ya que en ese caso y tal como decía en relación al proteccionismo, la tentación a free-ride el esfuerzo de los demás es muy grande. Sin embargo esta dificultad hay que sopesarla contra las ventajas que, en mi opinión poco o nada compartida, tiene la competencia entre jurisdicciones independientes, así como la importancia que ha tenido el principio de subsidiariedad a efectos de hacer posible la construcción del BCE, un argumento este que se encuentra también recogido en las publicaciones propias citadas en la nota 18.

En cuanto a España es cierto que la situación no es envidiable debido al tamaño relativamente grande de su burbuja inmobiliaria, a sus desequilibrios externos que han propiciado un mayor y más virulento contagio y a su falta de productividad se mida como se mida. En mi opinión y excepto en este último punto, nuestra economía se parece bastante a la de los EE.UU. En el aspecto del déficit exterior ambas economías, por las razones que acabo de subrayar, se encuentran en una situación muy parecida, aunque con una ventaja para España. El déficit exterior es, en efecto, sostenible siempre que el país tenga un sector financiero sólido que propicia la flight to quality. Este es, aparentemente, el caso de España y seguramente lo seguirá siendo a partir de la relativamente buena situación de su sector financiero y a la vista de una cierta consolidación de este sector que acabará llegando (con o sin Cajas) y que no es difícil de vislumbrar. Sin embargo, ya no es seguro que éste vuelva a ser el caso de los EE.UU. Por otro lado ese déficit exterior condiciona la necesaria promiscuidad para evitar los peores efectos del contagio y ahí hay que conceder una ventaja a la

${ }^{20}$ En este asunto hay que resaltar el esfuerzo realizado en VOXEU con relación a la consideración de esta problemática general de la apertura del comercio reuniendo a varios expertos bajo la dirección de Baldwin. Ver Baldwin y Evennet (2008)) 
posición estadounidense y la mala situación española a juzgar por los diferenciales que hay que pagar pata colocar la deuda española. Tampoco son estas dos economías tan disimilares en cuanto al mercado inmobiliario; pero aquí también me atrevo a ser herético y decir que las razones de fondo de la burbuja son más sostenibles aquí por razones demográficas europeas.

Desde luego que ambas economías se diferencian en cuanto a la innovación de todo tipo y a la productividad. Ya sabemos que esta es la asignatura pendiente de la economía española; pero aprobarla exige un cambio de mentalidad desde el conservadurismo a la toma de riesgos que no será fácil sin un cambio en la educación general. Pero esto yo no creo que llegue a verlo.

Creo que España se la juega en la audacia con que sepa encarar los retos de su situación específica. La prudencia no es hoy la mejor recomendación. Hace falta un plan audaz que tenga en cuenta los comentarios que acabo de poner en circulación. Solo así se podrán mejorar las expectativas y éstas son hoy y aquí cruciales. Pero esta actitud pone en riesgo la solvencia de la Banca y nuestro supervisor está obligado a recomendar y exigir prudencia. Un bonito problema de economía política.

Tomando como ejemplo a España se puede introducir ahora otro comentario de economía política que puede parecer tangencial pero que tiene intención constructiva y no poco recorrido. En efecto, en una situación como la actual, lo normal es que el Gobierno desee, por parte de la banca, una actitud arriesgada encaminada a maximizar ganancias pues esa actitud llevaría a incrementar las concesiones de crédito. Pero lo normal por parte de un supervisor cercano y conocedor de la situación concreta de cada institución, sea o no el Banco Central, sería la recomendación de prudencia para asegurar la supervivencia del sistema. Pues bien, si un banco se encuentra en medio de una crisis sistémica en una tesitura así, cabe pensar que un sistema de gobierno doble a la alemana podría ser adecuado. En efecto, el Consejo de Vigilancia podría ser el órgano adecuado para localizar la posición ideal entre la prudencia favorable a los accionistas y el conveniente arrojo a favor del sistema económico en general.

Para terminar con estos comentarios misceláneos me gustaría ofrecer una apostilla breve a la discusión abierta sobre del futuro de ese Capitalismo que hoy parece estar en entredicho. Casi desde agosto del 2007 han surgido los agoreros, desde los más tradicionales hasta los más futuristas, y se predice el final del sistema tal como lo conocemos, se vislumbra una nueva y más restrictiva regulación financiera y se augura una mayor presencia del Estado acompañada de un mayor tamaño del sector público. A mi juicio, sin embargo, de esta crisis surge al menos una buena noticia: se acabaron las ortodoxias de cualquier tipo. Si el nombre de Keynes está en boca de todo el mundo 
no debiera ser por la necesidad de movilizar el gasto público, sino por su rebeldía ante cualquier sabiduría convencional y su insistencia en encontrar la solución adecuada a cada situación sin dogmatismos. No es que Keynes haya resucitado tal como afirman asustados sus más fervientes críticos, o jalean los profetas del descalabro, sino que algunos enterraron al Keynes equivocado.

En efecto, Keynes hubiese dicho que, en las circunstancias actuales hay que tener mucho cuidado de no arrojar al niño con el agua del baño. En este sentido me parece provocador y muy relevante el comentario de Xabier Sala i Marti (2009) referido a nuestro país pero que puede utilizarse para poner en cuarentena los impulsos naturales de frenar la capacidad de innovación financiera: «Un micro-cosmos que refleja las ventajas e inconvenientes de la regulación lo tenemos en España, cuyo sistema financiero ha sido ampliamente alabado gracias a la rigidez impuesta por el Banco de España. Los reguladores impidieron a los bancos españoles comprar activos tóxicos, cosa que evitó el contagio procedente de Estados Unidos. Sin embargo, los reguladores no previeron que la crisis en España llegaría por otro lado y permitieron que los bancos se expusieran demasiado al sector inmobiliario, llegando a atarse de pies y manos a promociones inmobiliarias creadas en ciudades fantasmas. Es más, la extrema prudencia impuesta al sistema financiero hizo que la tasa de innovación en España fuera preocupantemente baja al no poder asumir los riesgos necesarios para financiar nuevas y arriesgadas tecnologías. Dicho de otro modo: si Sergei Brinn y Larry Page hubieran sido españoles, Google nunca hubiera sido una realidad porque ningún banco español hubiera financiado una idea tan arriesgada. España ha podido disfrutar de progreso tecnológico única y exclusivamente porque ese progreso tuvo lugar en el extranjero. Si no fuera por ello, España se encontraría anclada en el año 1970. Y si todo el mundo tuviera el sistema financiero español, quizá hubiéramos evitado la crisis de las subprime, pero el mundo entero estaría anclado»

\section{RESUMEN Y CONCLUSIÓN}

En este trabajo he tratado de ofrecer una explicación general de la naturaleza de la crisis, del caldo de cultivo del que surge, de su detonante y de las posibles políticas económicas que debieran aplicarse para sacamos de la situación en que nos encontramos. Para ello he acudido a ideas heterodoxa que he argüido están arrumbadas por pura «dependencia del recorrido». Estas ideas han sido recuperadas en el contexto de un modelo ortodoxo que, de hecho, no es sino un modelo de equilibrio general en miniatura. La parte heterodoxa del trabajo me parece que ofrece ciertas virtudes explicativas 
mientras que la parte ortodoxa permite aplicar ciertos resultados sobre el período de ajuste que merecerían un mayor desarrollo que, en cualquier caso, deberá quedar para otra ocasión.

De todas formas es obligatorio reconocer el trabajo de Caballero et al. (2008) en el que han tratado de ir al verdadero origen de la crisis. Comenzaré por mencionar sucintamente que se podría fijar el origen de la crisis no en la burbuja inmobiliaria y su acomodo por parte de la banca, sino en un tiempo anterior. Las crisis anteriores ya mencionadas en la nota 20 propiciaron una bajada sustancial de los tipos de interés de forma que la Banca tuvo que acostumbrarse a hacer beneficios a base de aumentar las inversiones. Esto les llevó simultáneamente a incrementar las hipotecas y a generar otros derivados a partir de esas hipotecas y de su titulación, derivados estos cuyo precio es difícil de fijar. Pero cabe preguntarse por qué y cómo los desequilibrios en los que cayó la economía abierta americana y otras influyeron en esa situación llevándola al borde del abismo. La explicación de Caballero (2009) incide sobre el exceso de demanda de activos de poco riesgo que acabó llevando necesariamente a un déficit por cuenta corriente de la economía estadounidense y, añado yo, de la economía española también, de forma que el tipo de interés real acabó siendo negativo. Quede este comentario aquí pues no cabe hacerle justicia en el espacio de este trabajo. Como tampoco cabe detenerse ante líneas prometedoras de investigación si queremos aprende algo teórico y general de esta crisis. Mencionaré tres. Hay que examinar la crisis en modelos neoclásicos de crecimiento y dinero en los que se especifique el mercado financiero como el de Urrutia (1975) ya mencionado, hay que extender esta modelización a situaciones de desequilibrio generalizado y hay que profundizar en el origen remoto de la crisis explorando la vía abierta por Caballero y otros (2008) o las ideas contenidas en el libro, próximo a aparecer, de V. Acharia y M. Richardson (2009)

Dejando pues aparte estas posibles extensiones de la vía que he seguido en este trabajo, termino diciendo que mi esperanza es que haya quedado clara la lección que hemos aprendido de acuerdo con los rasgos heterodoxos introducidos. Es imprescindible recuperar la confianza en el sistema de pagos conformado por los bancos para lo cual es inevitable distinguir los bancos contaminados de los que no lo están. Mientras esto no se haga los parches fiscales serán inútiles para restaurar la confianza en la capacidad y buen funcionamiento del sistema de pagos y, sin esta confianza, el sistema no fluye y las inyecciones de gasto público debilitan su efecto. O sea, que cuando y si el corredor neoclásico ha sido reducido a un estrecho senderito por un fallo de demanda efectiva como el que hoy vivimos, el mercado por sí mismo no arregla nada y el gasto público tampoco, aunque suaviza los efectos más desagradables de una crisis. Lo que hace falta es ensanchar ese pasillo neoclásico mediante una significativa ayuda a la concesión 
de crédito por parte de los bancos. Pero esto se puede hacer si el valor del colateral, propio o avalado, es grande, cosa que ya no es posible, bien por la caída de los precios de algunos activos que sí han sido flexibles, como las acciones cotizadas en Bolsa por ejemplo, bien porque los avales del Estado tienen sus límites si no queremos pasar a la nacionalización. Alternativamente solo cabe que los bancos estén dispuestos a arriesgarse a pesar del poco valor de ese colateral. Pero esto último depende de la confianza entre ellos mismos y ésta solo se recupera de verdad cuando existe algún mecanismo para poder identificar los especímenes sanos de los enfermos de forma que se evite el recelo generalizado sobre la salud del sector por parte de todo el mundo incluyendo los propios bancos. La clave está en recuperara la confianza.

\section{REFERENCIAS BIBLIOGRÁFICAS}

Acharia,V. y Richardson, M. (2009), Restoring Financial Stability: how to Repair a Failed System, Wiley, de publicación en marzo.

Arellano, M. y Bentolila, S. (2009), «La burbuja financiera: causas y responsables», Negocios, El País, domingo, 22 de febrero.

BALDWIn, R. y EvEnNet, S. (2008), What world leaders must do to halt the spread of protectionism, A Vox/EU.org publication, CEPR.

Bentolila, S. (2008), «Hay que levantar el veto a la reforma laboral en España», Sociedad Abierta, reproducido en inglés en VOXEU.

Barro, R. y Grossman, H. ( 1976), Money, Employment and Inflation, Cambridge University Press.

Blanchard, O. (2009a), «(Nearly) nothing to fear but fear itself», Ecomnomic Focus, The Economist, 29 de enero.

- (2009b), «Blanchard Round Table», The Economist, 30 de enero.

Caballero, R. J. (2009), «A global perspective on the great financial insurence run: Causes, consequences and solutions», Part1 and Part 2, VOXEU, 23 de enero de 2009.

Caballero, R. J.; Farhi, E. y Gourinchas, P.-O. (2008), «An Equilibrium Model of Global Imbalances and Low Interest Rates», American Economic Review, 98:1, págs. 358-393.

Dohtani, A.; Inaba, T. y HosaKa, H. (2007), «Corridor Stability of the Neoclassical Steady State», en T. Asada y T. Ishikikawa (eds.), Time and Space in Economics, Springer.

ECONOMIST.COM: http://www.economist.com

Felgueroso, F. y Jiménez, S. (2009), «Sobre crisis, retrasos y reforma laboral», Negocios, El País, domingo 22 de febrero.

Grauwe, P. de (2009), «Flexibility is out: now we see rigidity's virtues», Financial Times, lunes, 22 de febrero. 
Guerrieri, V. y Kondor, P. (2008), «Fund Managers,Career Concerns and Asset Price Volatility», no publicado, dirigirse a vguerrieri@ chicagogsb.edu.

Koвayashi, K. (2008a), «Subprime loans crisis. Lessons from Japana's Decade of Deception», no publicado pero descargable desde Kobayashi (2008b).

- (2008b), «Financial Crisis Management: Lessons from Japan’s Failures», VOXEU, 27 de octubre.

Krugman, P. (2008 y 2009), columnas semanales en el NYT, recogidas todas en Economist's View, http://economistsview.typepad.com/economistsview.

LaIDler, D. (2006), «Axel Leijonhufvud and the Quest for Microfoundations - Some reflections», UCLA, agosto, 30-31, http://farmer.sscnet.ucla.edu/NewWeb/testing/AxelConference/Papers/Laidler.pdf.

Leionhufvud, A. (1968), On Keynesian Economics and the Economicas of Keynes, Nueva York, Oxford University Press.

— (1973), «Effective demand failures», Sweedish Economic Journal 75:27-48.

— (2009), « No ordinary recession», en VOXEU: http://www.voxeu.org

MANKIw, G. (2008), «Spending and tax multipliers», http://gregmankiw.blogspot.com

Pesek, B. P. y Saving, T. R. (1967), Money, Wealth and Economic Theory, Londres, Macmillan.

Sala i Marti, X. (2008), «Crisis Financiera (3), Neo-Intervencionismo», La Vanguardia 17 de noviembre.

Rizzo, M. (2009), «Keynes Supported Counter-Cyclical Payroll Tax Reducctions», ThinkMarkets, http://thinkmarkets.wordpress.com.

Sargeant, T. J. (2008), «Evolution and Intelligent Design», American Economic Review 98(1), pág. 537.

SOCIEDAd AbIERTA: http://sociedadabierta.es.

Solow, R. (1956), «A Contribution to the Theory of Economic Growth», Quarterly Journal of Economics, 79, págs. 65-94

Spence, M. (1974) Market Signaling: Informational Transfer in Hiring and Related Screening Processes, Cambridge: Harvard University Press.

Spilimbergo, A.; Symansky, S.; Blanchard, O. y Cottrelli, C. (2008), Fiscal Policy for the Crisis, FMI.

Stiglitz, J. (1975), «The Theory of Screening, Education and Distribution of Income», American Economic Review, 65 (3), junio, págs. 283-300.

Snowdon, B. (2002), «Outside the Mainstream: An Interview with Axel Leijonhufvud», University of Northumbria,

Taleb, N. N. ( 2007), The Black Swan. The impact of the highly improbable, Random House.

URrutia, J. (1975), «Crecimiento, dinero y la configuración óptima de la economía», Cuadernos de Economía, septiembre-diciembre, págs. 514-542. 
Urrutia, J. (1982), «Curiosidades y paradojas en un modelo elemental de teoría neoclásica del crecimiento», Boletín de Estudios Económicos, XXXVII, núm. 119, págs. 367-384.

- (1984), «La influencia del sector público y de la distribución en la velocidad de ajuste a trayectorias optimas de crecimiento», Boletín de Estudios Económicos, XXXIX, núm. 121, págs. 201-209.

- (1997), «Una sugerencia para plantearse el problema de la reputación del Banco Central Europeo», Perspectivas del Sistema Financiero, 58, págs. 45-50.

- (2008a), «¿Qué hacemos? Un toque de heterodoxia», Expansión, 5 de febrero. Recogido en: juan. urrutiaelejalde.org

- (2008b), «El Corredor Neoclásico: Recordemos Japón», Expansión, 4 de marzo. Recogido en: juan.urrutiaelejalde.org

- (2008c), El Capitalismo que Viene, Planta 29.

VOXEU, http://www.voxeu.org/ 